\title{
Stability of clogging arches in a silo submitted to vertical vibrations
}

\author{
C. Lozano, * I. Zuriguel, and A. Garcimartín \\ Departamento de Física y Matemática Aplicada, Facultad de Ciencias, Universidad de Navarra, 31080 Pamplona, Spain \\ (Received 23 February 2015; published 5 June 2015)
}

\begin{abstract}
We present experimental results on the endurance of arches that block the outlet of a two-dimensional silo when subjected to vertical vibration. In a recent paper [C. Lozano et al., Phys. Rev. Lett. 109, 068001 (2012)], it was shown that the arch resistance against vibrations is determined by the maximum angle among those formed between each particle in the bridge and its two neighbors: the larger the maximum angle is, the weaker the bridge. It has also been reported that the breaking time distribution shows a power-law tail with an exponent that depends on the outlet size, the vibration intensity, and the load [I. Zuriguel et al., Sci. Rep. 4, 7324 (2014)]. Here we connect these previous works, demonstrating the importance of the maximum angle in the arch on the exponent of the breaking time distribution. Besides, we find that the acceleration needed to break an arch does not depend on the ramp rate of the applied acceleration, but it does depend on the outlet size above which the arch is formed. We also show that high frequencies of vibration reveal a change in the behavior of the arches that endure very long times. These arches have been identified as a subset with special geometrical features. Therefore, arches that cannot be broken by means of a given external excitation might exist.
\end{abstract}

DOI: 10.1103/PhysRevE.91.062203

PACS number(s): $45.70 . \mathrm{Ht}, 45.70 . \mathrm{Mg}$

\section{INTRODUCTION}

In recent years, substantial advancements have been achieved in the understanding of the physical mechanisms leading to clogging in the discharge of silos by gravity. It is known that if the outlet orifice is only a few times larger than the particles, the development of arches or domes may obstruct the orifice, completely arresting the flow [1]. Clog development has been shown to be memory independent, in the sense that no correlation is observed among consecutive avalanches [2] or in the initial positions in the silo of the particles that will end up forming the arch [3]. In addition, it has been shown that the clogging probability is constant during the entire duration of the avalanche, a result that is supported by the observation of an exponential decay in the avalanche size distribution [4-7]. This behavior-which has also been explained by a simple model [8]—-seems very robust. Indeed, deviations from it have only been found when anisotropy is introduced in the particles [4] or in the outlet geometry $[9,10]$. From the exponential decay of the avalanche size, the average can be calculated and used to investigate whether or not there exists a critical outlet size above which clogging will never occur. This question is still open [4,5] and, in a recent work, it has been proposed that there is no sharp discontinuity in the clogging behavior as the outlet size is enlarged, but only a strong increase of the relaxation times [11].

An alternative approach to the avalanche size measurements that may shed light on this problem is the study of the stability of clogging arches. This is usually investigated by supplying an external input of energy to the system, often in the form of a vibration. Despite the practical improvements that a good comprehension of this situation would bring, the number of fundamental works aiming to understand it is still scarce (see, however, Refs. [12-17]). From theses studies, it ensues that

\footnotetext{
*c.lozano@physik.uni-stuttgart.de; Present address: Max-PlanckInstitut für Intelligente Systeme - Heisenbergstraße 3, 70569 Stuttgart, Germany.
}

the flow in vibrated silos becomes intermittent as the orifice size decreases, alternating between flow and arrest intervals. The transition from flowing to arrested is controlled by the clogging (or arch formation) process, whereas the passing from arrested to flowing involves the unclogging (or arch destruction) process. Some results indicate that for low enough vibration amplitudes, the clogging process is not affected by the external excitation [13], which instead promotes arch destruction. A similar behavior was reported in [14] where, in addition, it was shown that the statistical distribution of breaking times (i.e., the time it takes to break an arch or, alternatively, the duration of the clog) exhibits a power-law tail. This result is still not well understood, even though it could be related to aging or creeping motion [18]. Regardless of its physical origin, the power-law distribution has a major consequence, as in some cases (small outlet size and low vibration intensity) its exponent may fall below two, and hence the first moment would not converge. Therefore, the average clog duration grows unboundedly with the reservoir size and measuring time, so the mean flow rate tends to zero, and the system is said to be in a clogged state (even though some grains may occasionally come out, they are statistically insignificant in the long term average) [16].

In that work, we showed that the behavior observed in granular materials flowing through a bottleneck can also be found in other systems, such as a model of pedestrians, a herd of sheep [19], and colloidal suspensions. In all cases, it was reported that the avalanche (or burst) size distribution decays exponentially, whereas the unclogging time displays a power-law tail $t^{-\alpha}$ whose exponent strongly depends on the system condition. This allowed us to sketch a state diagram for the flow with two states: a clogged one when $\alpha \leqslant 2$ and an unclogged one when $\alpha>2$. Three generic state variables were identified: (i) a length scale, (ii) the incompatible load, and (iii) the compatible load; the meaning of the latter two issues from the proposal by Cates et al. [20]. For the case of a silo, the specific variables would be (i) the aspect ratio between the outlet and the particle size, (ii) the intensity of the external excitation (which can be 
local or global), and (iii) the forces supported by the clogging structure.

In a related work, it was proved that the resistance of the clogging arch against vibrations is strongly determined by its morphology. In particular, it was found that the particle in the arch with the maximum associated angle $\phi_{\max }$ was, in most cases, the weakest position where it breaks [15]. This behavior was enhanced for arches with $\phi_{\max }>180^{\circ}$, i.e., a configuration called a defect, where a particle is gripped above its equator and hangs from its two neighbors. To endorse this idea, a simple model was proposed considering the forces acting on the defects, which was able to reproduce the experimentally found relationship between the maximum angle in the structure and the force needed to break it.

In this paper, we extend and connect the aforementioned works $[15,16]$. First, we will describe in detail the experimental setup. In the next section, we will characterize the observed arches, paying special attention to the defects. In another section, we will present the results regarding the acceleration at which arches collapse. We have investigated the effect of the vibration frequency, the outlet size, and the rate at which the acceleration is increased. The subsequent section is devoted to the study of the time that it takes for an arch to collapse when submitted to a constant acceleration. It will be shown that the maximum angle of the arch also determines its endurance in this scenario. In the last section, we will summarize our conclusions.

\section{EXPERIMENTAL PROCEDURE}

The experiments are performed using a two-dimensional silo placed on top of a shaker, as sketched in Fig. 1(a). The silo is made of two transparent polycarbonate panels $(390 \mathrm{~mm}$ high $\times 80 \mathrm{~mm}$ wide) coated with indium tin oxide (ITO) in order to prevent electrostatic charging. A thin frame, made of 1.2-mm-thick steel strips, is sandwiched between the two sheets. These metal strips constitute the side walls, so the distance between them defines the width of the reservoir (60 $\mathrm{mm}$ ). At the equator of the structure, two opposite metal flanges divide the silo into two equal containers $(180 \mathrm{~mm}$ high and $60 \mathrm{~mm}$ wide). The flanges leave between their inner edges an opening of size $R$ that can be changed at will [see inset of Fig. 1(a)]. The particles are monodisperse brass beads of diameter $d=1.00 \pm 0.01 \mathrm{~mm}$. As the gap between the two plates is $1.2 \mathrm{~mm}$, the particles can only arrange themselves in a monolayer. Note that although this cannot be strictly considered a two-dimensional disposition, the results obtained are not expected to be significantly different from such an ideal scenario [15]. An examination of the grain surface with an electron microscope revealed roughness in features with a size of a few microns or smaller.

The silo is placed on top of a TiraVib 52100 electromagnetic shaker capable of delivering a sinusoidal vertical acceleration of up to $10 \mathrm{~g}$ with a residual transversal noise below $15 \%$ of the vertical value. The shaker is commanded by a Stanford Research System DS345 function generator. The vibration is characterized by a measurement carried out with an accelerometer attached to the silo, whose signal is picked up by a HP54510 digitizing oscilloscope. A motor permits the silo to rotate automatically $180^{\circ}$ around

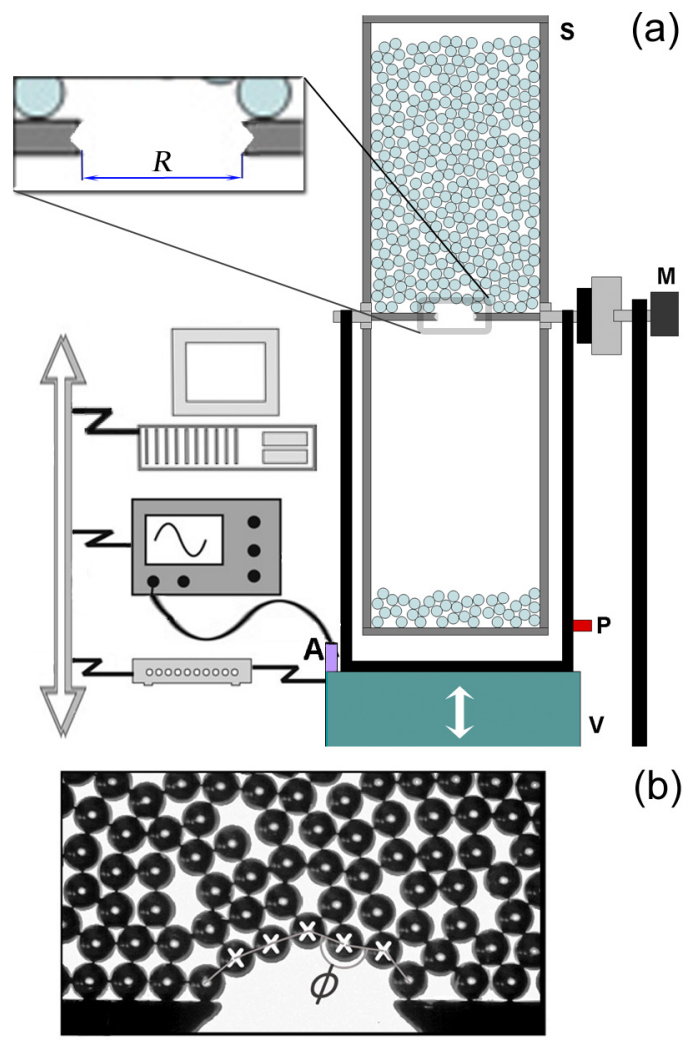

FIG. 1. (Color online) (a) Sketch of the experimental setup. A: accelerometer; V: electromagnetic shaker; M: motor; S: silo; P: proximity sensor; FG: function generator; O: oscilloscope; PC: computer. Top left inset: A magnification of the flanges forming the orifice of length $R$. The motor and the proximity sensor are also linked to the PC. (b) A photograph of the beads near the orifice (their centers are marked with dots) showing an arch (crosses and gray line) where the angle $\phi$ for one bead is also marked.

the horizontal axis (the vertical position is established by using a proximity sensor located at the bottom of the silo to stop the motor). Additionally, a CCD camera takes a picture of the region of interest, just above the orifice, every $45 \mathrm{~ms}$.

In order to collect a sizable amount of data, the entire measurement procedure is fully automated using a customized software written in LABVIEW. At the beginning of the measurement cycle, all of the beads are in the lower container of the silo. Then, the silo rotates half a turn and the particles start to flow from the upper container through the orifice until an arch blocks it, arresting the discharge. We detect the instant when the flow halts by comparing two successive frames obtained by the camera. To quantify the change between two snapshots, an image analysis is carried out to measure the absolute value of the difference. The threshold is established when this difference is $30 \%$ (if the grains are moving, the images are blurry and changing; in contrast, when the arch is formed, the pictures are sharp and unaltered). Then a snapshot of the region near the orifice, including the arch, is taken and stored in the computer [see Fig. 1(b)]. At this point, the shaker is switched on, producing a sinusoidal oscillation whose intensity 
is quantified by means of the adimensional acceleration $\Gamma$,

$$
\Gamma=\frac{A 4 \pi^{2} f^{2}}{g},
$$

where $A$ is the amplitude, $f$ is the frequency, and $g$ is the gravity acceleration. The breaking of the arch is again detected by comparing successive frames obtained by the camera. The breaking time measured in this way is stored in the computer together with the vibration parameters at which the arch collapses. Finally, the shaker produces a strong vibration to empty the upper container and the device is ready to restart the measurement cycle.

As the statistical analysis requires sets of arches developed under the same conditions, we have only considered those formed when the head of the grains above the orifice is higher than $90 \mathrm{~mm}$ (1.5 times the width of the silo). We estimate the head of grains from the time that the orifice has been flowing. We assume that in this case, Janssen saturation is achieved (see, for instance, [21]). We have recorded between 1000 and 7000 of such arches for each run.

To determine the stability of the arches, we implement two different experimental procedures. (i) When measuring the acceleration at which the arches collapse, we set a fixed frequency $f$ and the amplitude of the vibration $A$ is linearly increased with time (starting from zero) till the arch breaks down. In these conditions, the ramp rate chosen to increase the acceleration $(\Delta \Gamma / \Delta t)$ is a meaningful parameter that must be controlled. (ii) In order to measure the time that an arch resists against the external perturbation, the shaker supplies a vibration with a constant acceleration $\Gamma$. As the time measurement is obtained by comparing successive camera snapshots, the breaking time has a resolution of $45 \mathrm{~ms}$. Note that just $5 \mathrm{~ms}$ are attributable to computation, whereas $40 \mathrm{~ms}$ are due to the image acquisition rate. We have set beforehand an upper limit for the breaking time, $t_{\max }$, of $1200 \mathrm{~s}$. If an arch has not been broken after this time, the event is logged and the measurement protocol restarts.

\section{ARCH SHAPE}

First of all, we will consider the morphology of the clogging arches. They are defined as a set of mutually stabilized particles that span the whole width of the orifice and withstand the weight above them [22,23]. According to this definition, the mere removal of any particle in the arch would lead to the destabilization of the entire structure. Experimentally, we performed an image analysis of the arch pictures to accurately extract the position of the particles. From these positions, we identify the particles belonging to the clogging arch with the same protocol used in previous works $[15,24,25]$. In summary, we take into account the first line of beads that are just above the outlet. The grains touching the base of the silo are not considered part of the bridge as, in principle, they are stable by themselves [Fig. 1(b)]. An exception to this occurs when a particle at the end of an arch is ensnared at the wedge, so that its center lies inside the orifice opening. In this case, the particle is considered to be part of the arch because it would fall if any other of its particles is removed, thus fulfilling the aforementioned criterion.

It is known that clogging arches tend to exhibit a semicircular shape [1], a result nonetheless compatible with the existence of a rather high amount of local irregularities [24]. In particular, it was reported that the number of defects - particles that form an angle with their neighbors $\phi>180^{\circ}$-is about $17 \%$ in clogging arches obtained with stainless steel beads. The formation of defects was linked to frictional forces. Hidalgo et al. corroborated, using numerical simulations, that high values of the friction coefficient enable the formation of arches with larger $\phi$ [25]. These are associated with large values of tangential forces in the defects, where normal forces were surprisingly low. In our experiments, we checked that the percentage of defects within the arch is also about $17 \%$, even though the material used (brass) is different from [24].

We studied whether there is a preferred position for the defects along the arch; this is an issue that was not analyzed in preceding works and might have meaningful consequences. For a given orifice size, $R=4.50 \mathrm{~mm}$, we have calculated the frequency map of the position of the particles belonging to all the recorded arches that blocked the orifice, which is about 7500 [Fig. 2(a)]. The darker areas correspond to places where the probability to find a grain belonging to an arch is higher. We also calculated the normalized histogram of defects, i.e., particles that form an angle with their neighbors $\phi>180^{\circ}$ [Fig. 2(b)]. It can be seen that the picture is roughly similar, but if we compute the probability of finding a defect (number of defects divided by the number of beads belonging to an arch at a given place), we have found that it is not evenly distributed [Fig. 2(c)]. Defects are more likely at the lower zones of the distribution as, by definition, they are slightly downwards compared to the other beads in the arch. (a)

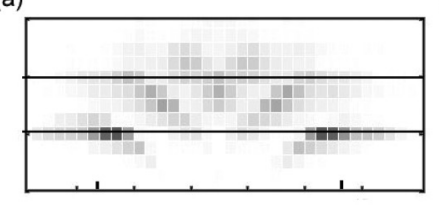

(b)

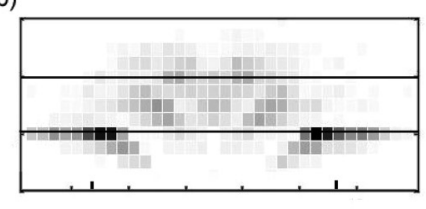

(c)

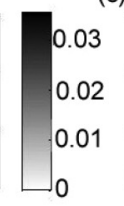

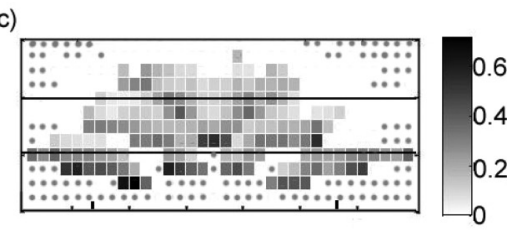

FIG. 2. (a) Frequency map of the position of the beads in the arches. (b) Frequency map of the position of the defects [the probability gray scale at the right of (b) is also valid for (a)]. (c) Relative probability of defects (number of defects divided by number of beads in each cell). Data correspond to the clogging arches recorded for $R=4.50 \mathrm{~mm}$. If the number of beads in a cell is less than 10 , the value obtained is refused due to the small number of events; in this case, the box is left white and marked with a gray dot. Horizontal lines are drawn as a guide for the eye at 1 and $2 \mathrm{~mm}$ height. The maps cover a region of $7 \times 3 \mathrm{~mm}$ (the horizontal axis is centered at the orifice; the vertical axis starts at the silo base; ticks are spaced $1 \mathrm{~mm}$ and two bigger ticks mark the borders of the orifice). 

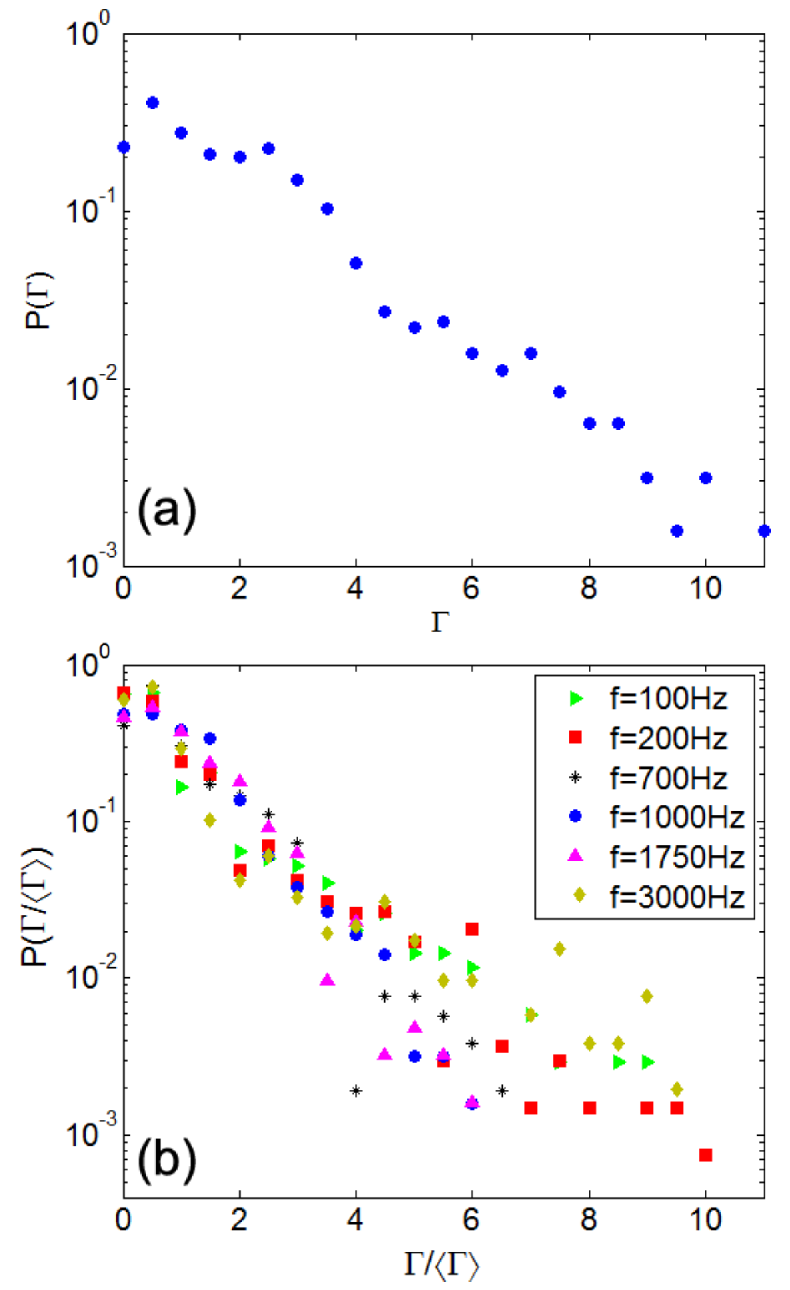

FIG. 3. (Color online) (a) PDF of the acceleration $\Gamma$ needed to break arches (results for $R=4.00 \mathrm{~mm}$ at $f=1000 \mathrm{~Hz}$ ). (b) PDF of the rescaled acceleration $\Gamma /\langle\Gamma\rangle$ needed to break arches (results for $R=4.00 \mathrm{~mm}$ at different frequencies $f$ as indicated in the legend). Note the semilogarithmic scale on both plots.

\section{BREAKING ACCELERATION}

In this section, we report the results concerning the acceleration needed to break the arches. As explained above, in each run of experiments we set the frequency and increase the vibration amplitude at a constant rate until the arch collapses. The number of realizations we perform for each set of parameters ( $R, f$, and amplitude ramp rate) is at least 1000 . In Fig. 3(a), we represent the probability density function of $\Gamma$ obtained for $R=4.00 \mathrm{~mm}, f=1000 \mathrm{~Hz}$, and a ramp rate of $0.09 \mathrm{~g} / \mathrm{s}$. Albeit noisy, an exponential decay is evidenced and, therefore, the average dimensionless acceleration $\langle\Gamma\rangle$ can be used as a characteristic parameter of the distribution. Hence, the distributions obtained under different conditions can be collapsed into a single curve just by rescaling $\Gamma$ with $\langle\Gamma\rangle$. This is shown in Fig. 3(b) for the outcomes obtained with an assortment of frequencies. The only noticeable difference between distributions is $\langle\Gamma\rangle$, which lies in the range $0.35<\langle\Gamma\rangle<1.80$ (see Table I).

The relatively small variation of $\langle\Gamma\rangle$ with $f$ implies that the amplitude at which the arches break, on the contrary, must
TABLE I. Mean acceleration needed to break arches at different frequencies (arches obtained for $R=4.00 \mathrm{~mm}$ ).

\begin{tabular}{lcccccccc}
\hline \hline$f(\mathrm{~Hz})$ & 80 & 100 & 200 & 500 & 700 & 1000 & 1750 & 3000 \\
\hline$\langle\Gamma\rangle$ & 0.38 & 0.4 & 0.35 & 0.78 & 1.07 & 1.80 & 1.03 & 0.76 \\
\hline \hline
\end{tabular}

be strongly dependent on the frequency. This is manifested in Fig. 4 (note the logarithmic scale). The dashed line represents the relationship $A \propto f^{-2}$, which would hold if $\langle\Gamma\rangle$ did not depend on $f$. (This line has been drawn for the mean value of $\langle\Gamma\rangle$ averaged over all the frequencies.) It follows that $\langle\Gamma\rangle$ is the best variable to quantify the stability of the arches, while $A$ is not a sound descriptor due to its strong dependence on frequency. For this reason, in the rest of this section, $\langle\Gamma\rangle$ will be used to quantify the effect of different parameters in the silo unclogging process. A fixed frequency is used in our tests; the effect of varying the frequency, which — as arguedis probably much smaller, is left for future works.

We have checked that within a certain range, the ramp rate at which the acceleration is increased has a negligible effect on the acceleration needed to break the arches. To this end, we have carried out several runs with different amplitude ramps (at constant rates of $0.045,0.09$, and $0.18 \mathrm{~g} / \mathrm{s}$ ) for a fixed frequency $f=1000 \mathrm{~Hz}$ and orifice size $R=4.45$ $\mathrm{mm}$. As evidenced by the outcomes shown in Table II, $\langle\Gamma\rangle$ is independent of the ramp rate, at least in the range explored. Of course, much slower or faster ramp rates might lead to a big change in the values of $\langle\Gamma\rangle$ if the dynamics of the phenomenon is modified; for instance, if the breaking probability changes with time (this issue will be considered in the next section).

A deeper insight into the effect of the ramp rate on the acceleration needed to break an arch can be gained by plotting $\langle\Gamma\rangle$ versus the maximum angle in the $\operatorname{arch}\left(\phi_{\max }\right)$ for different ramp rates, as shown in Fig. 5. It was previously reported that the larger the maximum angle is, the weaker the arch.

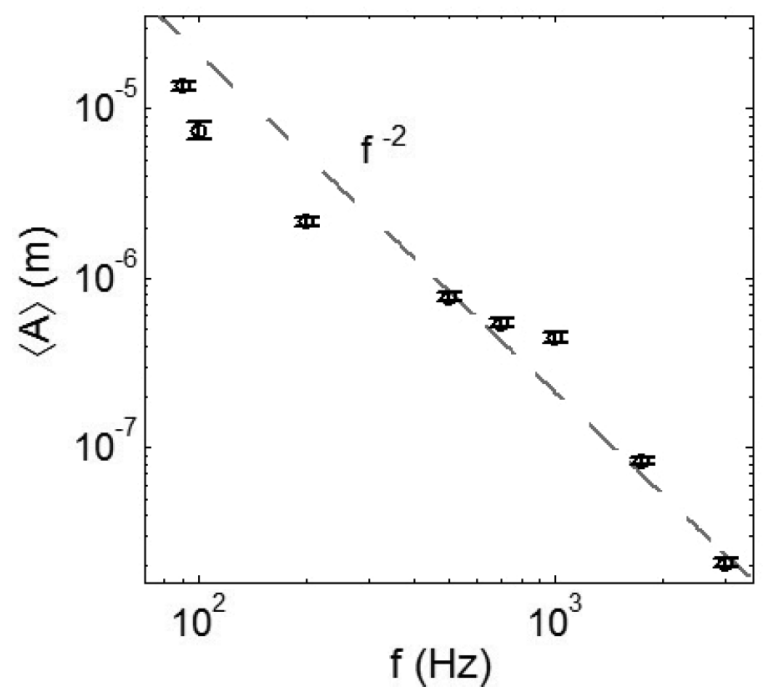

FIG. 4. The mean amplitude $A$ at which arches break, as a function of the frequency for $R=4.00 \mathrm{~mm}$, plotted in logarithmic scale. The dashed line represents Eq. (1) for a value of $\Gamma$ equal to the average over all of the frequencies. 
TABLE II. Mean acceleration at which arches break for different ramp rates (arches obtained for $R=4.45 \mathrm{~mm}$ ).

\begin{tabular}{lccc}
\hline \hline Ramp rate $(\mathrm{g} / \mathrm{s})$ & 0.045 & 0.09 & 0.18 \\
\hline$\langle\Gamma\rangle$ & $1.59 \pm 0.04$ & $1.57 \pm 0.04$ & $1.60 \pm 0.05$ \\
\hline \hline
\end{tabular}

This behavior is particularly marked for $\phi_{\max }>180^{\circ}$. In accordance with the average values presented in Table II, the ramp rate is shown to cause no significant effect on the dependence between $\langle\Gamma\rangle$ and $\phi_{\max }$.

Let us now investigate the stability of arches developed for different orifice sizes $R$. As expected, the larger the outlet is, the weaker the arches [Fig. 6(a)]. In principle, this effect could be attributed to the increasing size of the arches that is needed in order to provoke clogs with larger outlet sizes. The number of particles in clogging arches increases with $R$, and as a consequence the probability of finding larger values of $\phi$ in the arch is higher [15]. Then, in order to unveil any direct effect of the outlet size on the arch stability, one should compare arches with the same maximum angle. This is done in Fig. 6(b) where we plot $\langle\Gamma\rangle$ as a function of $\phi_{\max }$ for four different $R$. In all of the cases, $\langle\Gamma\rangle$ decreases as $\phi_{\max }$ grows, demonstrating the major dependence of the arch stability on the defects. But it can also be observed that the series for smaller $R$ are systematically a little above the ones obtained for larger $R$. This behavior is corroborated by the chart displayed in Fig. 6(c). In this graph, values of $\langle\Gamma\rangle$ for sets of arches defined by narrow intervals of $\phi_{\max }$ are plotted versus $R$. Clearly, there is a residual dependence of $\langle\Gamma\rangle$ on $R$ which cannot be attributed to the different probability of finding larger $\phi_{\max }$ in the arch (as revealed when considering arches with the same $\phi_{\max }$ for different $R$ ). We cannot presently provide an explanation for this effect.

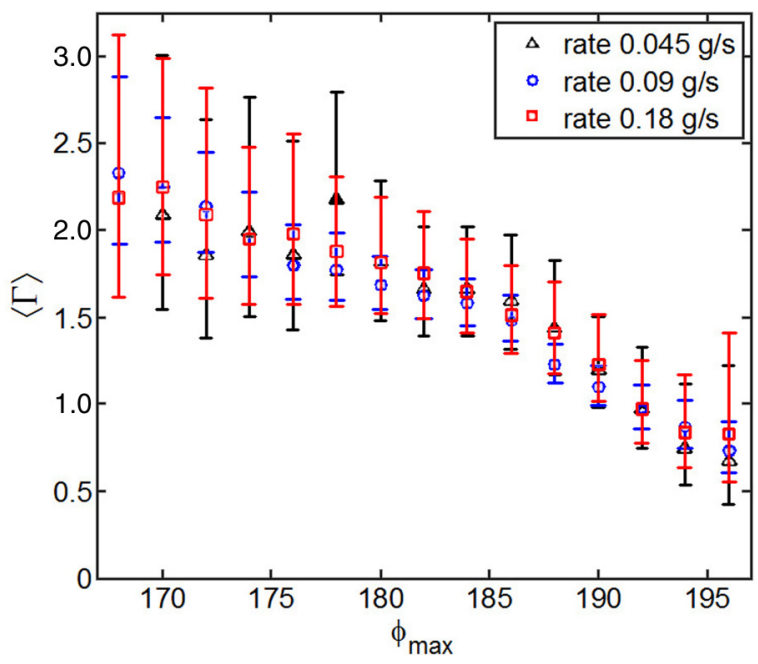

FIG. 5. (Color online) The mean acceleration $\Gamma$ at which arches break, as a function of the maximum angle in the $\operatorname{arch} \phi_{\max }$, for $f=$ $1000 \mathrm{~Hz}$ and $R=4.00 \mathrm{~mm}$, and different ramp rates, as indicated in the legend.

\section{BREAKING TIME}

In this section, we report the measurements of the time that it takes to break an arch when all of the experimental parameters (i.e., $R, \Gamma$, and $f$ ) are kept constant. As previously reported [14], the statistical distribution of breaking times $t_{b}$ suggests the existence of a power-law tail $t_{b}^{-\alpha}$ because its probability distribution function (PDF) displays approximately a straight line when plotted in logarithmic scale [Fig. 7(a)]. This is confirmed by representing the complementary cumulative distribution function (also called the survival or reliability function), which can be understood as the probability of finding an arch which endures the vibration for a time $T$ longer than $t_{b}$ [Fig. 7(b)]. Note that the slope of the complementary cumulative distribution function is $\alpha+1$. The fittings performed to obtain the exponent of the power-law tail $(\alpha)$ are carried out with the Clauset-Shalizi-Newman method [26], which provides the value of $\alpha$ and the lower limit for the breaking time $t_{b(\min )}$ from which the fit is valid, along with an estimation of the goodness of the fit (a $p$-value). In Fig. 7(c), such survival distributions are plotted along with the fitting curves for several outlet sizes. As expected, increasing $R$ leads to a corresponding increase of $\alpha$ [Fig. 7(d)], as the probability of finding very large values of $t_{b}$ becomes smaller. Interestingly, for the experimental conditions of Fig. 7(c), a transition is observed from distributions where the first moment converges $(\alpha>2)$ to distributions where the first moment does not converge $(\alpha \leqslant 2)$, a general scenario recently described [16]. In the latter case, the average increases unboundedly with the number of measurements (or maximum measuring time) as it is dominated by a few extreme events (meaning very large occurrences found at the end of the tail).

With the aim of shedding light on the nature of the arches that lead to such long breaking times, we have focused on one of the distributions whose first moment does not converge ( $R=4.50 \mathrm{~mm}$ ). For this instance, we recorded the rupture of more than 7000 bridges. We have grouped the data in several intervals according to the maximum angle in the arch, and we have plotted the complementary cumulative distributions of $t_{b}$ for each group. Although the quality of the fits for some of the categories becomes poorer, due to the smaller number of events in the subsets, the outcomes shown in Fig. 8(a) reveal a strong dependence of $\alpha$ on $\phi_{\max }$ : the larger $\phi_{\max }$, the higher $\alpha$ is [Fig. 8(b)]. As a result, arches with more important defects are weaker in terms of the time that they resist against a vibration. This matches the result reported in the previous section that inversely related the strength of the arch (quantified by the acceleration at which it breaks) to $\phi_{\max }$. Remarkably, among all of the distributions of breaking times [Fig. 8(a)], only the one corresponding to arches with $\phi_{\max } \in\left[172^{\circ}, 176^{\circ}\right]$ has a nonconvergent first moment. This indicates that the nonconvergence is linked to the influence of very regular arches, which are more likely to endure the external perturbation for longer times. Irregular arches, on the other hand, always lead to distributions whose first moment $\left\langle t_{b}\right\rangle$ converges. If this mean is plotted as a function of the maximum angle in the arch [Fig. 8(c)], a sudden increase of $\left\langle t_{b}\right\rangle$ is observed as $\phi_{\max }$ decreases, revealing the major role of this variable in the stability of arches. In this graph, the vertical dashed line indicates the value of $\phi_{\max }$ for which the first 

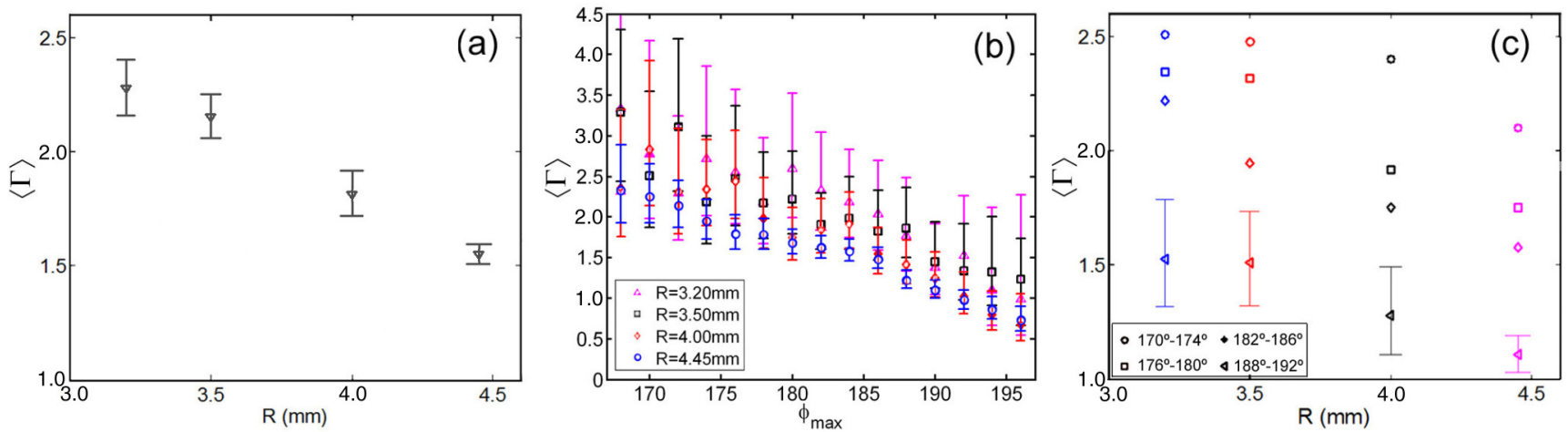

FIG. 6. (Color online) (a) Average acceleration $\langle\Gamma\rangle$ at which arches break as a function of the outlet size $R$. (b) Acceleration $\langle\Gamma\rangle$ at which arches break, as a function of the maximum angle in the arch $\phi_{\max }$, for different values of $R$, as indicated in the legend. (c) $\langle\Gamma\rangle$ vs $R$ for small intervals of $\phi_{\max }$. Error bars indicate $95 \%$ confidence intervals.
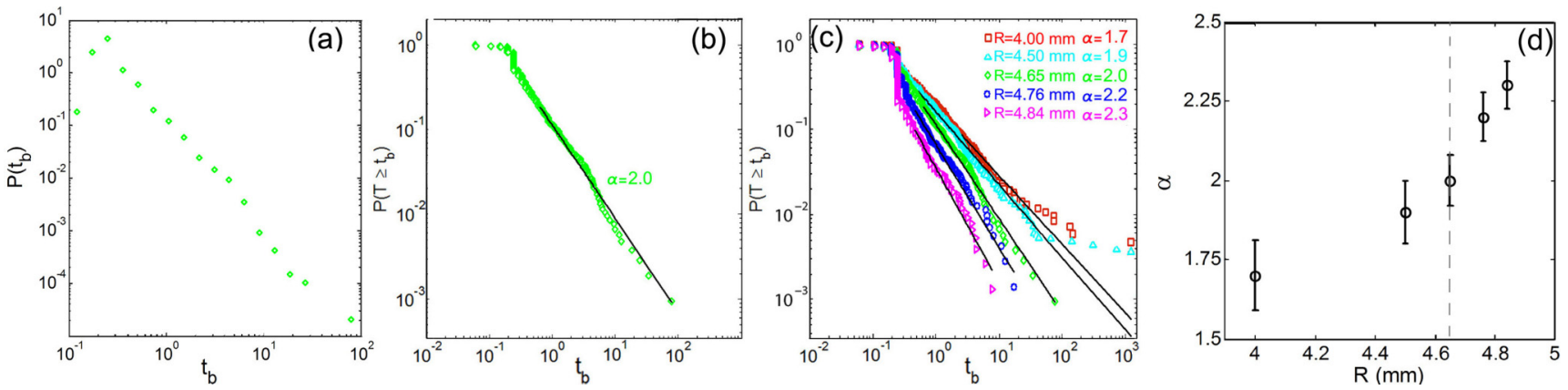

FIG. 7. (Color online) (a) Logarithmic plot of the breaking times $t_{b}$ histogram obtained for $R=4.65 \mathrm{~mm}, \Gamma=2.6, f=100 \mathrm{~Hz}$. Bin size is logarithmic. (b) Complementary cumulative distribution function (also called survival or reliability function) of $t_{b}$ corresponding to the same data used in (a). The solid line is a fit performed with the method described by Clauset et al. [26]. (c) Complementary cumulative distribution functions of $t_{b}$ for $\Gamma=2.6$ and $f=100 \mathrm{~Hz}$ with different orifice sizes $R$, as indicated in the legend along with the respective exponents $\alpha$ calculated from the fits (solid lines). (d) The exponent $\alpha$ of the power-law tail as a function of the outlet size $R$. The vertical dashed line indicates the value below which the first moment of the distribution does not converge.
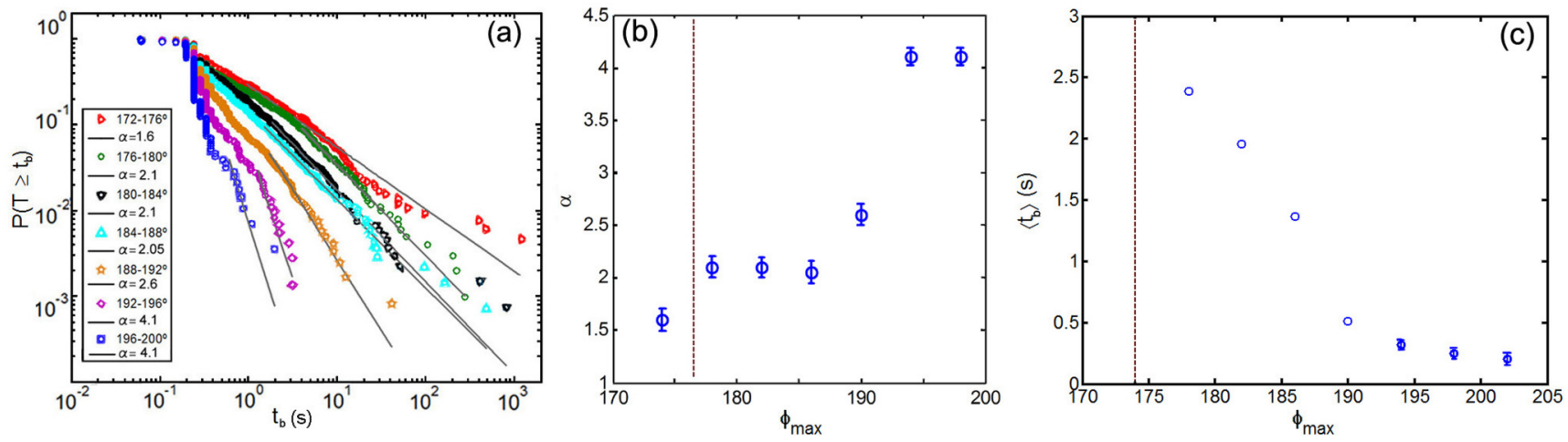

FIG. 8. (Color online) (a) Complementary cumulative distribution function of $t_{b}$ for $R=4.50 \mathrm{~mm}, \Gamma=2.6$, and $f=100 \mathrm{~Hz}$ corresponding to small intervals of the maximum angle in the $\operatorname{arch} \phi_{\max }$, as indicated in the legend along with the values of $\alpha$. Solid lines are fits (see [26]). (b) The exponent $\alpha$ of the power-law tail as a function of $\phi_{\max }$. The vertical dashed line indicates the value below which the first moment does not converge. The error bar in $\alpha$ is smaller than the size of the symbol. (c) The average breaking time at which arches break $\left\langle t_{b}\right\rangle$ as a function of $\phi_{\max }$. Note that the second moment (used to calculate the error bars) does not converge below a certain value of $\phi_{\max }$. The vertical dashed line indicates the value below which the first moment does not converge. 


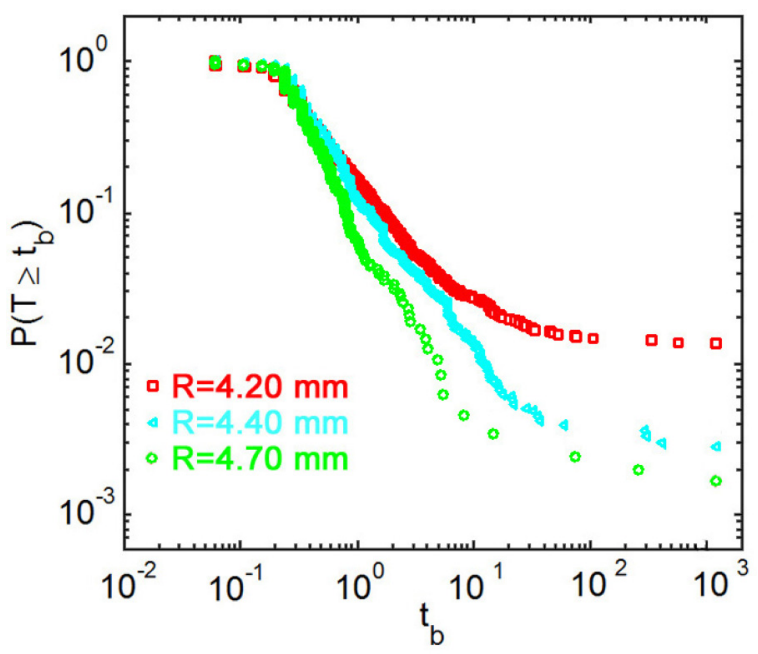

FIG. 9. (Color online) Complementary cumulative distribution function of $t_{b}$ obtained for $\Gamma=5.0$ at $f=1000 \mathrm{~Hz}$ and different $R$, as indicated in the legend.

moment of the distribution does not converge (and therefore the average cannot be rigourously calculated).

Finally, let us focus on a salient feature that, in some circumstances, is apparent in the survival distribution. For instance, in Fig. 7(c), it can be noticed that the results obtained for small $R$ (which at the same time have smaller exponent) deviate towards higher values of $P\left(T \geqslant t_{b}\right)$ than the power-law fits. This occurs for large values of $t_{b}$ (in this particular case, for arches that resist more that around 100 seconds). This departure from $t_{b}^{-\alpha}$ suggests that other mechanisms might be at play. In order to check the robustness of this behavior, we have performed experiments at a higher frequency $(f=1000 \mathrm{~Hz}$ instead of $100 \mathrm{~Hz}$ ), with correspondingly smaller amplitudes for a given acceleration $\Gamma$. The outcomes (displayed in Fig. 9) reveal even stronger deviations from the power-law distributions, which start at about 10 seconds. Indeed, for the smallest value of $R$, the curve becomes almost flat after $t_{b} \approx 100 \mathrm{sec}$, implying that the arches that have not been destroyed after 100 seconds will probably survive more than 1000 seconds (of course, just a small fraction of arches will be so robust: about 1 in 100 for $R=4.20 \mathrm{~mm}$ ). This behavior supports the statement that for some conditions of the external perturbation, there can exist some arches that will last forever (in the sense described above). In other words, for some parameters of the external vibration, it is not warranted that the flow will be eventually restored in a finite amount of time.

\section{CONCLUSIONS}

We have analyzed the procedure to unclog a silo by applying an external perturbation in the form of a vertical vibration. The viewpoint we have taken to do it is to consider the individual arches that block the exit. We have studied their shape, taking cues from a previous work where we proved that geometrical defects are the most salient features that compromise the endurance of these structures against an external perturbation [15]. The defects - which are quite evenly distributed along the arches - are suitably quantified through the maximum angle $\phi_{\max }$. We have first considered the vibration intensity at which the arches break down when a linearly increasing acceleration (a ramp) is applied. In this respect, we have extended previous research and showed that the ramp rate has a negligible effect on the average value needed to break arches. In addition, we evidenced that the outlet size does have a residual influence on the acceleration at which arches collapse; and this is beyond the fact that the bigger the orifice, the more likely it is to find a defect in the arch that clogs it.

We have produced results along a different line by measuring the time it takes to break an arch under a constant vertical vibration. The importance of $\phi_{\max }$ on the arch endurance in this case has also been proved. We find that arches with large maximum angles collapse more quickly than those whose maximum angle is smaller, as one could have expected. More importantly, the distributions of the breaking times reveal power-law tails in which the first moment may not converge, depending on the conditions. Thus, large outlet sizes lead to breaking time distributions with a well-defined average, whereas small outlet sizes give rise to an ill-defined breaking time average. Of course, the mean value of a finite sample can always be calculated, but the figure will depend on the size sample (measuring time or number of experiments). This is so because the events at the far end of the tail contribute disproportionately to the average. We have progressed further and identified a relationship between arch shape and the existence of fat tails, which has been associated with regular arches where geometrical imperfections are very small. By taking a large statistical distribution (for a given outlet size) and splitting the whole sample of arches into several subsets chosen after their maximum angle, one realizes that the breaking time distributions for arches with large $\phi_{\max }$ have a well-defined average, whereas arches with smaller $\phi_{\max }$ have an ill-defined breaking time average. From this, it can be concluded that a subset of very regular arches, which are difficult to break up, provokes in some instances the transition to a clogged state where the average flow vanishes in the long term.

\section{ACKNOWLEDGMENTS}

We thank D. Maza, R. Cruz Hidalgo and G. Lumay for discussions and suggestions, and L. F. Urrea for technical help. This work has been financially supported by Ministerio de Economía y Competitividad (Spanish Government) through Projects No. FIS2011-26675 and No. FIS2014-57325, and Universidad de Navarra (PIUNA Project). C.L. thanks the Asociación de Amigos de la Universidad de Navarra for support through a scholarship.
[1] K. To, P.-Y. Lai, and H. K. Pak, Jamming of granular flow in a two-dimensional hopper, Phys. Rev. Lett. 86, 71 (2001).
[2] I. Zuriguel, L. A. Pugnaloni, A. Garcimartín, and D. Maza, Jamming during the discharge of grains from a silo described as a percolating transition, Phys. Rev. E 68, 030301(R) (2003). 
[3] J. Tang and R. P. Behringer, How granular materials jam in a hopper, Chaos 21, 041107 (2011).

[4] I. Zuriguel, A. Garcimartín, D. Maza, L. A. Pugnaloni, and J. M. Pastor, Jamming during the discharge of granular matter from a silo, Phys. Rev. E 71, 051303 (2005).

[5] K. To, Jamming transition in two-dimensional hoppers and silos, Phys. Rev. E 71, 060301(R) (2005).

[6] C. C. Thomas and D. J. Durian, Geometry dependence of the clogging transition in tilted hoppers, Phys. Rev. E 87, 052201 (2013).

[7] I. Zuriguel, A. Janda, A. Garcimartín, C. Lozano, R. Arévalo, and D. Maza, Silo Clogging Reduction by the Presence of an Obstacle, Phys. Rev. Lett. 107, 278001 (2011).

[8] T. Masuda, K. Nishinari, and A. Schadschneider, Critical bottleneck size for jamless particle flows in two dimensions, Phys. Rev. Lett. 112, 138701 (2014).

[9] S. Saraf and S. V. Franklin, Power-law flow statistics in anisometric (wedge) hoppers, Phys. Rev. E 83, 030301(R) (2011).

[10] S. Mondal and M. M. Sharma, Role of flying buttresses in the jamming of granular matter through multiple rectangular outlets, Granular Matter 16, 125 (2014).

[11] C. C. Thomas, D. J. Durian, Fraction of clogging configurations sampled by granular hopper flow, Phys. Rev. Lett. 114, 178001 (2015).

[12] J. R. Valdés and J. C. Santamarina, Clogging: bridge formation and vibration-based destabilization, Can. Geotech. J. 45, 177 (2008).

[13] C. Mankoc, A. Garcimartín, I. Zuriguel, D. Maza, and L. A. Pugnaloni, Role of vibrations in the jamming and unjamming of grains discharging from a silo, Phys. Rev. E 80, 011309 (2009).

[14] A. Janda, D. Maza, A. Garcimartín, E. Kolb, J. Lanuza, and E. Clément, Unjamming a granular hopper by vibration, Europhys. Lett. 87, 24002 (2009).

[15] C. Lozano, G. Lumay, I. Zuriguel, R. C. Hidalgo, and A. Garcimartín, Breaking Arches with Vibrations: The Role of Defects, Phys. Rev. Lett. 109, 068001 (2012).
[16] I. Zuriguel, D. R. Parisi, R. C. Hidalgo, C. Lozano, A. Janda, P. A. Gago, J. P. Peralta, L. M. Ferrer, L. A. Pugnaloni, E. Clément, D. Maza, I. Pagonabarraga, and A. Garcimartín, Clogging transition of many-particle systems flowing through bottlenecks, Sci. Rep. 4, 7324 (2014).

[17] P. A. Gago, D. R. Parisi, and L. A. Pugnaloni, "Faster Is Slower" Effect in Granular Flows, in Traffic and Granular Flow '11, edited by V. V. Kozlov, A. P. Buslaev, A. S. Bugaev, M. V. Yashina, A. Schadschneider, and M. Schreckenberg (Springer, Heidelberg, 2013), pp. 317-324.

[18] B. Blanc, J.-C. Géminard, and L. A. Pugnaloni, On-and-off dynamics of a creeping frictional system, Europhys. J. E 37, 112 (2014).

[19] A. Garcimartín, J. M. Pastor, L. M. Ferrer, J. J. Ramos, C. Martín-Gómez, and I. Zuriguel, Flow and clogging of a sheep herd passing through a bottleneck, Phys. Rev. E 91, 022808 (2015).

[20] M. E. Cates, J.-P. Wittmer, J.-P. Bouchaud, and P. Claudin, Jamming, Force Chains, and Fragile Matter, Phys. Rev. Lett. 81, 1841 (1998).

[21] C. Perge, M. A. Aguirre, P. A. Gago, L. A. Pugnaloni, D. Le Tourneau, and J.-C. Géminard, Evolution of pressure profiles during the discharge of a silo, Phys. Rev. E 85, 021303 (2012).

[22] A. Mehta, Spatial, dynamical and spatiotemporal heterogeneities in granular media, Soft Matter 6, 2875 (2010).

[23] L. A. Pugnaloni, G. C. Barker, and A. Mehta, Multi-particle structures in non-sequentially reorganized hard sphere deposits, Adv. Complex Syst. 4, 289 (2001).

[24] A. Garcimartín, I. Zuriguel, L. A. Pugnaloni, and A. Janda, Shape of jamming arches in two-dimensional deposits of granular materials, Phys. Rev. E 82, 031306 (2010).

[25] R. C. Hidalgo, C. Lozano, I. Zuriguel, and A. Garcimartín, Force analysis of clogging arches in a silo, Granular Matter 15, 841 (2013).

[26] A. Clauset, C. R. Shalizi, and M. E. Newman, PowerLaw Distributions in Empirical Data, SIAM Rev. 51, 661 (2009). 